\title{
Resectable Intrahepatic Cholangiocarcinoma
}

National Cancer Institute

\section{Source}

National Cancer Institute. Resectable Intrahepatic Cholangiocarcinoma. NCI Thesaurus.

Code C156908.

Intrahepatic cholangiocarcinoma that is amenable to surgical resection. 